\title{
ESTUDO DO PROCESSO DE LIXIVIAÇÃO DE CHUMBO DE CRTs, UTILIZANDO PLANEJAMENTO DE EXPERIMENTOS
}

\author{
U. M. PAPPIS ${ }^{1}$, C. M. dos REIS ${ }^{1}$, G. P. MELLO ${ }^{1}$, J. K. ALBIERO ${ }^{1}$, F. de CASTILHOS ${ }^{1}$ e D. A. \\ BERTUOL $^{1}$ \\ ${ }^{1}$ Universidade Federal de Santa Maria, Departamento de Engenharia Química \\ E-mail para contato: uilianpappis@gmail.com
}

\begin{abstract}
RESUMO - Com crescente avanço da tecnologia, é imprescindível que as técnicas de reciclagem de resíduos eletrônicos sejam aprimoradas. Este trabalho tem como objetivo a obtenção de um modelo que relacione as principais variáveis do processo de lixiviação de chumbo, com auxílio de planejamento experimental. O material a ser lixiviado é proveniente de tubos de raios catódicos, de televisores descartados. Após passar por um processo de moagem e separação granulométrica, o material foi submetido a uma lixiviação com ácido nítrico em diferentes condições. Um planejamento experimental com 4 variáveis, sendo elas, temperatura, concentração do ácido, relação sólido/líquido e tamanho de partícula, a 5 níveis, foi realizado para avaliar a influência de cada uma dessas variáveis no processo de lixiviação. Num tempo de 10 minutos observou-se que, para este planejamento, os termos linear e quadrático do tamanho de partícula, e linear da temperatura foram significativos no modelo obtido. $\mathrm{O}$ uso do planejamento de experimentos possibilitou determinar as variáveis que mais influenciam no processo.
\end{abstract}

\section{INTRODUÇÃO}

O crescimento desenfreado de novas tecnologias vem trazendo consigo um aumento considerável na quantidade de rejeitos eletrônicos. Dentre estes rejeitos estão os objetos deste estudo: os tubos de raios catódicos (CRT's), provenientes de televisores e monitores obsoletos. Segundo Elshkaki et al. (2005), de uma perspectiva global, estima-se que apenas 26,75\% dos CRT's descartados são reciclados, sendo que 59\% são colocados em aterros e 14,75\% são incinerados.

Estudos vêm sendo feitos para extrair o chumbo contido neste material, que representa $8 \%$ da sua massa total (Smith et al., 1996). Jha et al. (2012) estudaram a influência de diferentes ácidos, concentrações, agitações, temperaturas, tempos e relações sólido/líquido para lixiviação de chumbo de placas de circuito impresso. Já estudos realizados por Strzalkowska et al. (2012) mostraram a influência do tamanho de partícula e da concentração de ácido na lixiviação de CRT's, obtendo entretanto, rendimentos abaixo de $1 \%$. Com base nestes estudos, os principais fatores que influenciam o processo de lixiviação de chumbo são identificados, porém o impacto que cada variável tem no processo ainda não é quantificado.

Técnicas de planejamento de experimentos são comumente utilizadas para análise e modelagem 


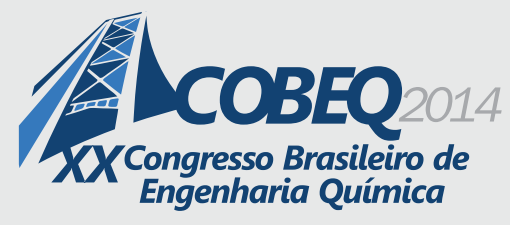

19 a 22 de outubro de 2014
Florianópolis/SC

de processos em várias áreas, pois elas definem em que quantidade e condições devem ser realizados os experimentos para coleta de dados mais eficiente, desta forma, impactando diretamente nos custos e tempo da pesquisa. Neste contexto, o principal objetivo deste estudo é utilizar planejamento de experimentos para investigar e quantificar os efeitos da temperatura, concentração do ácido, relação sólido/líquido e diâmetro de partícula na recuperação do chumbo através de lixiviação ácida.

\section{EXPERIMENTAL}

\subsection{Materiais e métodos}

Manualmente um aparelho de televisão foi desmontado, separando-se o CRT do restante. Do CRT ainda retirou-se a parte frontal, pois esta não contém chumbo (Menad, 1999). A parte cônica, que contém chumbo em forma de óxido, foi colocada em um moinho de bolas, transformando-se em pó. Com este, realizou-se uma separação granulométrica, utilizando peneiras de 200, 250, 325 e 400 mesh (Série Tyler), de modo a obter faixas de tamanho de partículas diferentes. O material retido em cada uma das três últimas peneiras foi analisado em equipamento de fluorescência de raios-X, para determinar a fração mássica de $\mathrm{PbO}$ na mistura antes da lixiviação (peneira 250 mesh - 24,48\%; peneira 325 mesh - 25,70\%; e peneira 400 mesh - 25,66\%), e com base nisso calcular o rendimento da extração Os valores das composições foram condizentes com os trabalhos de Méar et al. (2006) e Yuan et al. (2012).

Para realizar as lixiviações, foi utilizado um balão volumétrico com aquecimento, ligado a uma serpentina para condensação dos gases. A quantidade de ácido nítrico foi mantida constante em 100 $\mathrm{mL}$, variando a quantidade de sólidos, conforme a relação sólido/líquido. Com agitação magnética de $600 \mathrm{rpm}$, cada experimento teve duração de 10 minutos. A solução lixiviada foi filtrada e diluída a $250 \mathrm{~mL}$ com água deionizada em balão volumétrico. A análise da quantidade de chumbo recuperada foi feita em equipamento de absorção atômica Agilent Technologies 200 Series AA.

\subsection{Planejamento experimental}

Usualmente, estudos na área de recuperação de metais utilizam o método fatorial simples, variando uma variável com o tempo, e mantendo demais constantes, entretanto esse método requer muitos experimentos e é difícil de considerar as interações entre as diferentes variáveis (Brereton, 2003). O planejamento do tipo fatorial completo requer muitos experimentos (Box e Wilson, 1951) e o meio fatorial é aplicável se for conhecida a inexistência de interações entre as variáveis (Box e Hunter, 1961). Uma alternativa é o planejamento composto central desenvolvido por Box e Wilson, e aperfeiçoada por Box e Hunter, que fornece quase a mesma informação do planejamento fatorial completo, mas requer um número bem menor de experimentos (Box e Hunter, 1978).

Neste estudo foi utilizado o planejamento do tipo "Central Composite Design" (CCD), com os experimentos distribuídos simetricamente em torno do ponto central 0 , conforme Tabela 1. As variáveis estudadas neste trabalho foram temperatura $\left(\mathrm{X}_{1}\right)$, concentração de ácido nítrico $\left(\mathrm{X}_{2}\right)$, relação sólido/líquido $\left(\mathrm{X}_{3}\right)$ e o tamanho de partícula, representado pela malha da peneira na qual ficou retido o material na separação granulométrica $\left(\mathrm{X}_{4}\right)$. 
Tabela 1 - Níveis e fatores experimentais investigados

\begin{tabular}{|l|c|c|c|c|c|}
\hline \multirow{2}{*}{\multicolumn{1}{|c|}{ Fator }} & \multicolumn{5}{c|}{ Valores dos parâmetros } \\
\cline { 2 - 6 } & $\mathbf{- 2}$ & $\mathbf{- 1}$ & $\mathbf{0}$ & $\mathbf{+ 1}$ & $\mathbf{+ 2}$ \\
\hline $\mathrm{X}_{1}$ : Temperatura $\left({ }^{\circ} \mathrm{C}\right)$ & 35 & 50 & 65 & 80 & 95 \\
\hline $\mathrm{X}_{2}$ : Concentração $\mathrm{HNO}_{3}(\mathrm{M})$ & 0,1 & 0,2 & 0,3 & 0,4 & 0,5 \\
\hline $\mathrm{X}_{3}$ : Relação S/L $(\mathrm{w} / \mathrm{v})$ & $1 / 100$ & $3 / 100$ & $5 / 100$ & $7 / 100$ & $9 / 100$ \\
\hline $\mathrm{X}_{4}$ : Malha da peneira (mesh) & & 250 & 325 & 400 & \\
\hline
\end{tabular}

Foram incluídos $2^{\mathrm{n}}\left(2^{4}=16\right)$ pontos fatoriais, $2 \mathrm{n}(2 \times 4=8)$ pontos axiais, e 3 réplicas no ponto central, totalizando 27 experimentos. Estes foram realizados de forma aleatória, para evitar tendências.

A resposta $\left(\mathrm{Y}_{\text {exp }}\right)$ foi aproximada por um modelo polinomial incluindo um termo independente, quatro termos lineares, quatro termos quadráticos e seis termos de relação cruzada, conforme Equação 1.

$$
\begin{array}{r}
Y_{\text {mod }}=b_{0}+b_{1} X_{1}+b_{2} X_{2}+b_{3} X_{3}+b_{4} X_{4}+b_{11} X_{1}^{2}+b_{22} X_{2}^{2}+b_{33} X_{3}^{2}+b_{44} X_{4}^{2}+b_{12} X_{1} X_{2}+b_{13} X_{1} X_{3} \\
+b_{14} X_{1} X_{4}+b_{23} X_{2} X_{3}+b_{24} X_{1} X_{4}+b_{34} X_{3} X_{4}
\end{array}
$$

\section{RESULTADOS}

O planejamento utilizado e o rendimento de cada experimento são apresentados na tabela 2. Os rendimentos obtidos se mostraram satisfatórios, visto que foram superiores aos obtidos em condições semelhantes por Strzalkowska et al. (2012). Observa-se que o desvio padrão das triplicatas no ponto central foi de apenas $0,055 \%$, conferindo confiabilidade aos dados experimentais.

Após terem sido realizados os experimentos fizeram-se análises estatísticas para saber quais variáveis podem ser consideradas estatisticamente significativas. Como mostrado na Tabela 3, o termo linear da temperatura, e os termos linear e quadrático do tamanho de partícula se mostraram estatisticamente significativos, pois apresentam $\mathrm{p}<0,05$.

Os coeficientes do modelo polinomial da Equação 1, assim como o p valor são apresentados na tabela 4. O erro padrão é o termo que mede a variação em torno do valor estimado pelo modelo, e $t$ representa a taxa entre o coeficiente do modelo pelo seu erro padrão. A Equação 2 representa os dados com $95 \%$ de confiança.

$$
Y_{\text {mod }}=3,982407+0,387917 X_{1}+0,833333 X_{4}-0,155556 X_{4}^{2}
$$


Tabela 2 - Planejamento composto central

\begin{tabular}{|c|c|c|c|c|c|}
\hline Experimento & $\mathbf{X}_{1}$ & $\mathbf{X}_{2}$ & $\mathbf{X}_{\mathbf{3}}$ & $\mathbf{X}_{4}$ & $Y_{\exp }(\%)$ \\
\hline 1 & -1 & -1 & -1 & -1 & 2,75 \\
\hline 2 & -1 & -1 & -1 & 1 & 4,27 \\
\hline 3 & -1 & -1 & 1 & -1 & 2,35 \\
\hline 4 & -1 & -1 & 1 & 1 & 4,21 \\
\hline 5 & -1 & 1 & -1 & -1 & 2,65 \\
\hline 6 & -1 & 1 & -1 & 1 & 4,25 \\
\hline 7 & -1 & 1 & 1 & -1 & 2,43 \\
\hline 8 & -1 & 1 & 1 & 1 & 4,24 \\
\hline 9 & 1 & -1 & -1 & -1 & 3,48 \\
\hline 10 & 1 & -1 & -1 & 1 & 5,19 \\
\hline 11 & 1 & -1 & 1 & -1 & 3,19 \\
\hline 12 & 1 & -1 & 1 & 1 & 5,00 \\
\hline 13 & 1 & 1 & -1 & -1 & 3,52 \\
\hline 14 & 1 & 1 & -1 & 1 & 5,01 \\
\hline 15 & 1 & 1 & 1 & -1 & 3,46 \\
\hline 16 & 1 & 1 & 1 & 1 & 5,11 \\
\hline 17 & -2 & 0 & 0 & 0 & 3,34 \\
\hline 18 & 2 & 0 & 0 & 0 & 4,59 \\
\hline 19 & 0 & -2 & 0 & 0 & 3,72 \\
\hline 20 & 0 & 2 & 0 & 0 & 4,20 \\
\hline 21 & 0 & 0 & -2 & 0 & 3,96 \\
\hline 22 & 0 & 0 & 2 & 0 & 3,97 \\
\hline 23 & 0 & 0 & 0 & -1 & 3,03 \\
\hline 24 & 0 & 0 & 0 & 1 & 4,58 \\
\hline $25(C)$ & 0 & 0 & 0 & 0 & 3,99 \\
\hline $26(C)$ & 0 & 0 & 0 & 0 & 3,94 \\
\hline $27(C)$ & 0 & 0 & 0 & 0 & 4,05 \\
\hline
\end{tabular}

O modelo da Equação 2 representa os dados de forma satisfatória, visto que apresentou o valor para $R^{2}(\operatorname{adj})=97,46 \%$, enquanto o modelo que inclui todas as variáveis, inclusive as não significativas, apresentou valor para $\mathrm{R}^{2}=98,83 \%$. Verifica-se que o $\mathrm{R}^{2}$ considerando todas as variáveis não é muito maior, o que confirma que a temperatura e tamanho de partícula são realmente as variáveis significativas. Ou seja, acrescentar as demais não aumenta de forma significativa o $\mathrm{R}^{2}$. $\mathrm{Na}$ Figura 1 pode-se notar os valores observados em relação aos valores preditos, bem como o Gráfico de Pareto com a relevância de cada termo do modelo. 
Tabela 3 - Análise da variância para $\mathrm{Y}_{\text {mod }}(\mathrm{ANOVA})$

\begin{tabular}{|c|c|c|c|c|c|}
\hline Fator & $\mathbf{S S}^{\mathbf{a}}$ & $\mathbf{d f}^{\mathbf{b}}$ & $\mathbf{M S}^{\mathbf{c}}$ & $\mathbf{F}^{\mathbf{d}}$ & $\mathbf{p}$ valor \\
\hline $\mathbf{X}_{\mathbf{1}}$ & 3,6115 & 1 & 3,6115 & 222,0316 & 0,0000 \\
\hline $\mathbf{X}_{\mathbf{1}}^{\mathbf{2}}$ & 0,0002 & 1 & 0,0002 & 0,0132 & 0,9106 \\
\hline $\mathbf{X}_{\mathbf{2}}$ & 0,0590 & 1 & 0,0590 & 3,6275 & 0,0811 \\
\hline $\mathbf{X}_{\mathbf{2}}^{2}$ & 0,0004 & 1 & 0,0004 & 0,0265 & 0,8734 \\
\hline $\mathbf{X}_{\mathbf{3}}$ & 0,0513 & 1 & 0,0513 & 3,1562 & 0,1010 \\
\hline $\mathbf{X}_{\mathbf{3}}^{2}$ & 0,0002 & 1 & 0,0002 & 0,0132 & 0,9106 \\
\hline $\mathbf{X}_{\mathbf{4}}$ & 12,5000 & 1 & 12,5000 & 768,4873 & 0,0000 \\
\hline $\mathbf{X}_{\mathbf{4}}^{2}$ & 0,1452 & 1 & 0,1452 & 8,9258 & 0,0113 \\
\hline $\mathbf{X}_{\mathbf{1}} \mathbf{X}_{\mathbf{2}}$ & 0,0039 & 1 & 0,0039 & 0,2402 & 0,6329 \\
\hline $\mathbf{X}_{\mathbf{1}} \mathbf{X}_{\mathbf{3}}$ & 0,0039 & 1 & 0,0039 & 0,2402 & 0,6329 \\
\hline $\mathbf{X}_{\mathbf{1}} \mathbf{X}_{\mathbf{4}}$ & 0,0011 & 1 & 0,0011 & 0,0649 & 0,8032 \\
\hline $\mathbf{X}_{\mathbf{2}} \mathbf{X}_{\mathbf{3}}$ & 0,0352 & 1 & 0,0352 & 2,1614 & 0,1672 \\
\hline $\mathbf{X}_{2} \mathbf{X}_{\mathbf{4}}$ & 0,0077 & 1 & 0,0077 & 0,4707 & 0,5057 \\
\hline $\mathbf{X}_{3} \mathbf{X}_{\mathbf{4}}$ & 0,0410 & 1 & 0,0410 & 2,5210 & 0,1383 \\
\hline Erro & 0,1952 & 12 & & & \\
\hline Total SS & 16,6555 & 26 & & & \\
\hline
\end{tabular}

${ }^{\mathrm{a}}$ Soma dos quadrados, ${ }^{\mathrm{b}}$ Graus de liberdade, ${ }^{\mathrm{c}}$ Média dos quadrados, ${ }^{\mathrm{d}}$ Teste F

Tabela 4 - Coeficientes de regressão estimados.

\begin{tabular}{|c|c|c|c|c|}
\hline Fator & Teste T & p valor & Coeficiente & Erro Padrão \\
\hline Constante & 61,3256 & 0,6329 & 3,9824 & 0,0649 \\
\hline $\mathbf{X}_{\mathbf{1}}$ & 14,9007 & 0,0000 & 0,3879 & 0,0260 \\
\hline $\mathbf{X}_{\mathbf{1}}^{2}$ & $-0,1147$ & 0,9106 & $-0,0030$ & 0,0260 \\
\hline $\mathbf{X}_{\mathbf{2}}$ & 1,9046 & 0,0811 & 0,0496 & 0,0260 \\
\hline $\mathbf{X}_{\mathbf{2}}^{\mathbf{2}}$ & $-0,1627$ & 0,8734 & $-0,0042$ & 0,0260 \\
\hline $\mathbf{X}_{\mathbf{3}}$ & $-1,7766$ & 0,1010 & $-0,0463$ & 0,0260 \\
\hline $\mathbf{X}_{\mathbf{3}}^{\mathbf{2}}$ & $-0,1147$ & 0,9106 & $-0,0030$ & 0,0260 \\
\hline $\mathbf{X}_{\mathbf{4}}$ & 27,7216 & 0,0000 & 0,8333 & 0,0301 \\
\hline $\mathbf{X}_{\mathbf{2}}^{\mathbf{2}}$ & $-2,9876$ & 0,1013 & $-0,1556$ & 0,0521 \\
\hline $\mathbf{X}_{\mathbf{1}} \mathbf{X}_{\mathbf{2}}$ & 0,4901 & 0,6329 & 0,0156 & 0,0319 \\
\hline $\mathbf{X}_{\mathbf{1}} \mathbf{X}_{\mathbf{3}}$ & 0,4901 & 0,6329 & 0,0156 & 0,0319 \\
\hline $\mathbf{X}_{\mathbf{1}} \mathbf{X}_{\mathbf{4}}$ & $-0,2548$ & 0,8032 & $-0,0081$ & 0,0319 \\
\hline $\mathbf{X}_{\mathbf{2}} \mathbf{X}_{\mathbf{3}}$ & 1,4702 & 0,1672 & 0,0469 & 0,0319 \\
\hline $\mathbf{X}_{\mathbf{2}} \mathbf{X}_{\mathbf{4}}$ & $-0,6861$ & 0,5057 & $-0,0219$ & 0,0319 \\
\hline $\mathbf{X}_{\mathbf{3}} \mathbf{X}_{\mathbf{4}}$ & 1,5878 & 0,1383 & 0,0506 & 0,0319 \\
\hline
\end{tabular}




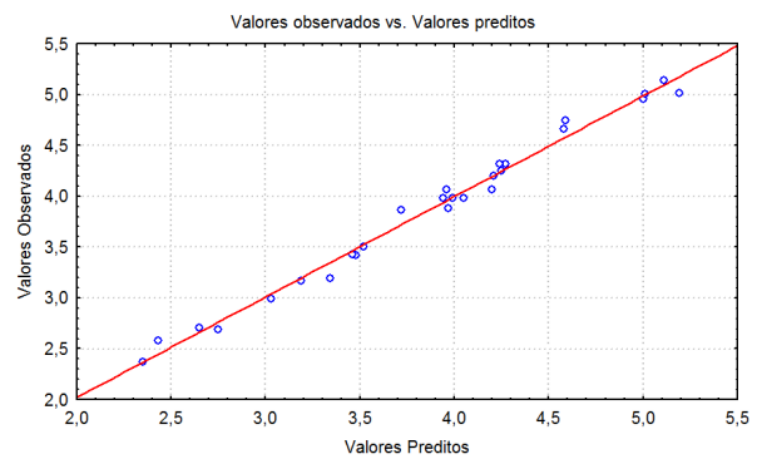

(a)

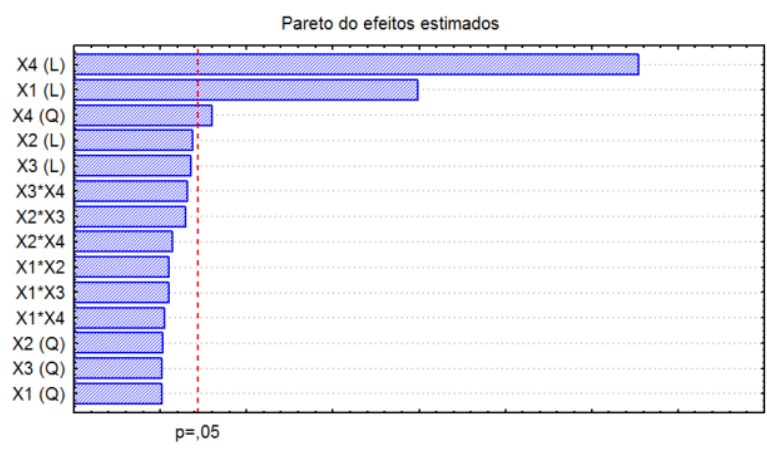

(b)

Figura 1 - Relação entre valores observados e preditos (a) e Gráfico de Pareto (b).

Os valores residuais, ou seja, o desvio do modelo em relação aos dados experimentais, conforme Equação 3, e o histograma dos mesmos, são apresentados na Figura 2. Observa-se que são valores muitos pequenos, e que grande maioria se posiciona no centro do histograma, aproximando-se do zero.

$$
\text { Resíduo }=\left(Y_{\text {experimental }}-Y_{\text {modelo }}\right)
$$

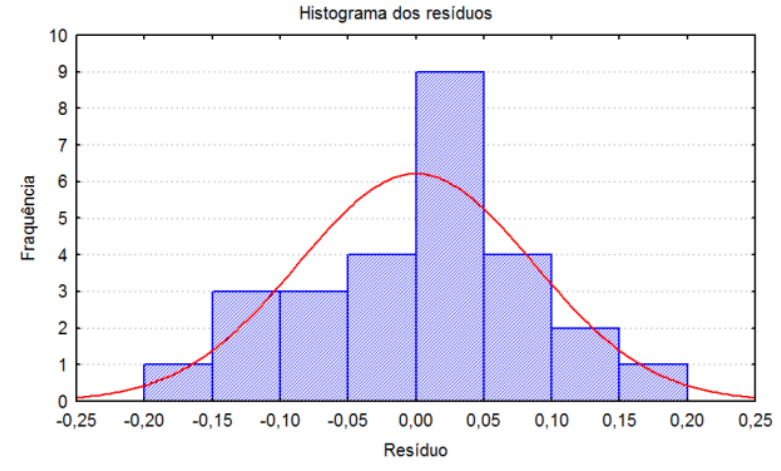

(a)

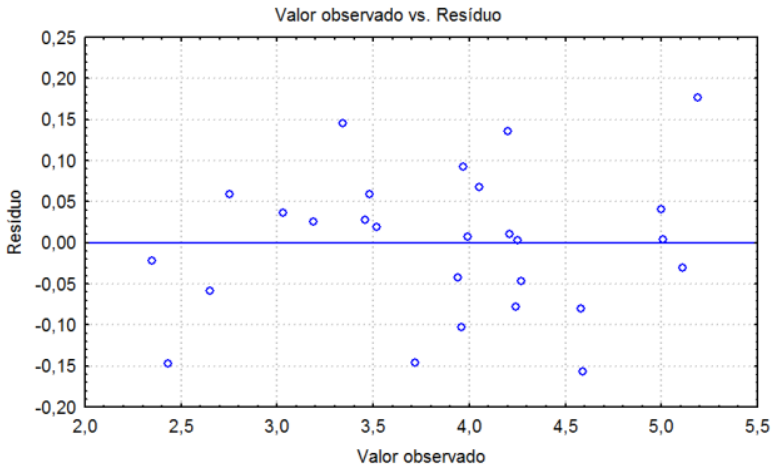

(b)

Figura 2 - Histograma dos resíduos (a) e resíduos em relação ao valor observado (b).

A fim de ter uma melhor visualização dos efeitos do modelo da Equação 2, a Figura 3 apresenta a superfície de resposta do mesmo. Observa-se, portanto, que um menor tamanho de partícula leva a um aumento na área superficial do material a ser lixiviado, permitindo maior contato com a solução lixiviante e facilitando a reação do metal de interesse. Da mesma forma, o aumento da temperatura também ocasionou um maior rendimento, que pode ter sido motivado por um possível aumento da difusividade do metal e/ou da solução lixiviante no sistema em função do acréscimo de energia. A concentração do ácido e a relação sólido/líquido não se mostraram tão significativas no modelo, pois 
as a temperatura e tamanho de partícula tiveram uma relevância muito maior, encobrindo os efeitos daquelas, como pode se observar no pareto da Figura 1.

Nas faixas estudadas, o ponto ótimo pode ser encontrado no menor tamanho de partícula e maior temperatura. Essas condições são válidas para valores estendidos, até a temperatura de ebulição do ácido, e menor tamanho de partícula obtido na etapa de moagem.

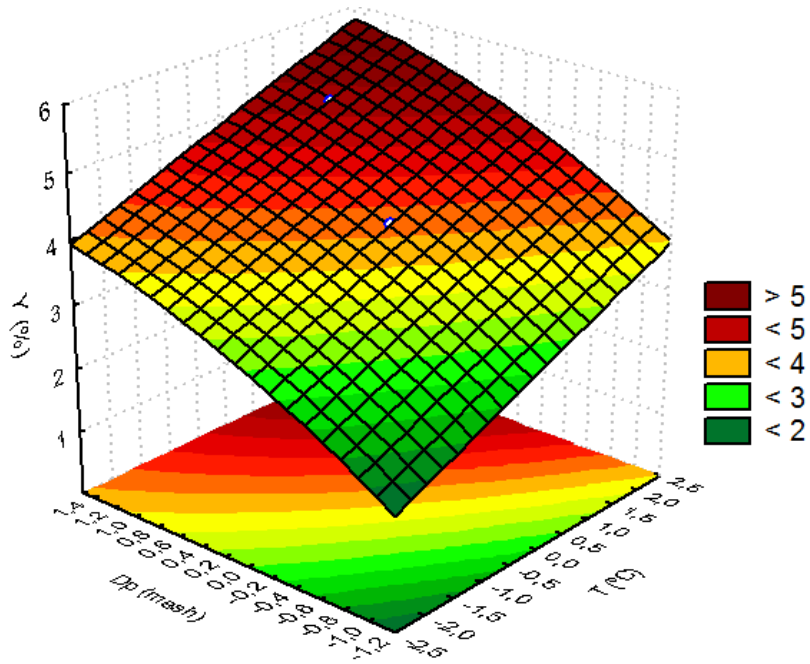

Figura 3 - Superfície de resposta do modelo para Y em função da temperatura e do tamanho de partícula.

\section{CONCLUSÕES}

Neste trabalho, o processo de lixiviação ácida de CRT's foi investigado com auxílio da técnica de planejamento de experimentos. As variáveis investigadas foram temperatura, razão sólido/líquido, tamanho de partícula e concentração de $\mathrm{HNO}_{3}$. Os valores de rendimento obtidos nas várias condições experimentais foram superiores aos valores reportados na literatura.

Com o planejamento de experimentos utilizado, foi possível encontrar quais são as variáveis mais significativas no processo de lixiviação ácida de CRT's, para um tempo de 10 minutos. Um modelo foi desenvolvido para o processo relacionando as variáveis envolvidas. Os resultados indicaram que o aumento da temperatura acarreta um aumento no rendimento da extração, pois apresenta o seu termo positivo no modelo. Para o tamanho de partícula obteve-se um termo negativo no modelo, significando que aumentando a malha da peneira (ou diminuindo o tamanho de partícula), aumenta-se o rendimento. $\mathrm{O}$ ajuste do modelo aos dados experimentais foi satisfatório. 


\section{REFERÊNCIAS}

ATRZALKOWSKA, A.; WOJTALA, M.; SIWKA, J. Pb(II) leaching from waste CRT funnel glass in nitric acid solutions, JAMME, v. 55/2, p. 825-828, 2012.

BRERETON, R. G. Data Analysis for the Laboratory and Chemical Plant. Ed. John Wiley and Sons, p.15-19, 2003.

BOX, G. E. P.; WILSON, K. B. On the Experimental Attainment of Optimum Conditions. J. R. Stat. Soc., Series B, 13, 1-45, 1951.

BOX, G. E. P.; HUNTER. The $2^{\mathrm{k}-\mathrm{p}}$ Fractional Factorial Designs. J. S., J. Technometrics. v. 3, p. 311458, 1961.

BOX, G. E. P.; HUNTER, W. G.; HUNTER, J.S. Statistics For Experimenters: An Introduction to Design, Data Analysis and Model Building. New York: Ed John Wiley, 1978.

ELSHKAKI, A.; VOET, V. D. E.; TIMMERMANS, V.; HOLDERBEKE, M. V. Dynamic stock modeling: a method for the identification and estimation of future waste streams and emissions based on past production and product stock characteristics. Energy v. 30, p. 1353-63, 2005.

JHA, M.K.; KUMARI, A.; CHOUBEY, P.K.; LEE, J.; KUMAR, V.; JEONG, J.. Leaching of lead from solder material of waste printed circuit boards (PCBs). Hydrometallurgy, v. 121-124, p. 28-34, 2012.

MÉAR, F.; YOT, P.; CAMBOM, M.; RIBES, M. The characterization of waste cathode-ray tube glass. Waste Manage, v. 26, p. 1468-1476, 2006.

MENAD, N. Cathode ray tube recycling. Resour Conserv Recy, v. 26, p. 143-154, 1999.

SMITH, D.; SMALL, M.; DODDS, R.; AMAGAI, S.; STRONG, T. Computer recycling: a case study. Eng. Sci. Educ., v. 5(4) 159 - 64, 1996.

YUAN, W.; LI, J.; ZHANG, Q.; SAITO, F. Innovated application of mechanical activation to separate lead from scrap cathode ray tube funnel glass. Environ. Sci. Technol., v. 46, p. 4109-4114, 2012. 